\title{
Influence of Reducing Agents on Biosafety and Biocompatibility of Gold Nanoparticles
}

\author{
Dong-Lin Xia • Yu-Fei Wang • Ning Bao • Hong He • \\ Xiao-dong Li • Yan-Pei Chen $\cdot$ Hai-Ying Gu
}

Received: 1 April 2014 / Accepted: 24 July 2014 /

Published online: 30 September 2014

(C) Springer Science+Business Media New York 2014

\begin{abstract}
Extensive biomedical applications of nanoparticles are mainly determined by their safety and compatibility in biological systems. The aim of this study was to compare the biosafety and biocompatibility of gold nanoparticles (GNPs) prepared with HEPES buffer, which is popular for cell culture, and sodium citrate, a frequent reducing agent. From experimental results on the body weight and organ coefficients of acute oral toxicity tests, it could be observed that HEPES-prepared GNPs are biologically safer than citric-prepared GNPs at the same dose of $500 \mu \mathrm{g} / \mathrm{kg}$. The in vitro cell viability was higher for HEPES-prepared GNPs than citric-prepared GNPs at 5.0- and $10.0-\mathrm{ug} / \mathrm{mL}$ concentrations. More reactive oxygen species (ROS) were generated in the cell suspension when supplemented with citric-prepared GNPs than HEPES-prepared GNPs when their concentrations were higher than $20 \mu \mathrm{g} / \mathrm{mL}$. The results stated that HEPES-prepared GNPs had better biosafety and biocompatibility than citric-prepared GNPs. This study not only revealed the influence of reducing agent on biosafety and biocompatibility of nanomaterials but also provided accumulative evidence for nanomaterials in biomedical applications.
\end{abstract}

Keywords Biosafety $\cdot$ Biocompatibility $\cdot$ Gold nanoparticles $\cdot$ Reducing agent $\cdot$ HEPES

Dong-Lin Xia and Yu-Fei Wang contributed equally to this work.

D.-L. Xia $\cdot$ Y.-F. Wang $\cdot$ N. Bao $・$ X.-d. Li $\cdot$ Y.-P. Chen $\cdot$ H.-Y. Gu

School of Public Health, Nantong University, Nantong 226019, China

D.-L. Xia • Y.-F. Wang • N. Bao $・$ H.-Y. Gu

Institute of Analytical Chemistry for Life Science, Nantong University, Nantong 226019, China

H. He

Affiliated Hospital of Nantong University, Nantong 226001, China

H.-Y. Gu ( ()

Nantong University, 9 Seyuan Road, 226019 Nantong, Jiangsu, People's Republic of China e-mail: hygu@ntu.edu.cn

H.-Y. Gu

e-mail: guhy99@21cn.com 


\section{Introduction}

Biosafety and biocompatibility are critical factors for almost all biological and medical applications. In 1997, International Standard ISO-10993 was formulated in order to provide a comprehensive and general guideline on biocompatibility of materials for medical applications, with priority on cell culture-based in vitro tests. For modern biotechnology, biosafety should be guaranteed so that the potential adverse impact could be minimized and managed in a manner without a negative impact on biodiversity and human health. The investigation on the biosafety of nanomaterials could effectively eliminate doubts from the public. More importantly, quantitative study of the biocompatibility of nanoparticles is fundamental for us to understand their interactions with biological systems. Therefore, biosafety and biocompatibility of nanomaterials have attracted more and more attention during the past years $[1,2]$.

Among a number of nanomaterials, gold nanoparticles (GNPs) have shown tremendous growth in various biomedical applications because of their convenient preparation, controllable sizes, and easy modification. Extensive uses of GNPs make it necessary and inevitable to assess their biosafety and biocompatibility [3]. Previous studies had revealed that gold ions were toxic on some types of cells, such as red blood cells [4]. In vivo studies revealed that the toxicity of GNPs was detectable in hematology, blood chemistry, and inflammatory and pathological response [5-8]. It is necessary to improve the safety of GNPs to avoid harming the human body.

More evidences have to be accumulated on the toxicity of GNPs for in vivo and in vitro applications. Previous investigations have revealed that the biosafety of GNPs was likely dependent on their composition, size, and surface characteristics [9, 10]. Semmler-Behnke et al. observed that a considerable percentage of GNPs with the size of $18 \mathrm{~nm}$ could be removed from the blood and trapped predominantly in the liver and spleen [11]. GNPs could be taken up by Kupffer cells in the liver or by macrophages in other places, regardless of the particle size [12].

In the preparation of GNPs, especially reducing agents could reduce gold ions and stable GNPs. In the past, sodium citrate has been commonly utilized as the reducing agent. Under physiological circumstances, however, citrate-prepared GNPs could form aggregates easily $[13,14]$. In a biological system, this condition might be even worse since high-level accumulation of GNPs in liver and spleen might induce gene mutations and liver necrosis [15]. To date, various approaches on the fabrication of GNPs have been developed with the aim to improve their biocompatibility and biosafety for in vivo and in vitro applications [16, 17]. Proteins, peptides, DNA, and other chemical/biological polymers have been used to prepare GNPs in order to improve their biocompatibility [18]. Such studies suggested that surface modification could be essential for safe utilization of GNPs. Therefore, it might be possible to improve the biocompatibility of nanoparticles by using new reducing reagents [18].

Since many kinds of nanoparticles could interact with cells, it is of importance to ensure that their modifications do not cause adverse effects. It is desirable to develop biocompatible and green ways for preparing GNPs. Herein we studied the influence of reducing agents on the biosafety and biocompatibility of gold nanoparticles; we chose 2-[4-(2-hydroxyethyl)-1piperazinyl] ethanesulfonic acid (HEPES), which is a popular buffer extensively applied for cell culture [19], and a commonly used sodium citrate as reducing agents. Our experimental results showed that HEPES-prepared GNPs were normally safer in acute toxicity studies under in vivo and in vitro conditions. It is estimated that our study could potentially extend applications of GNPs in biomedical areas. 


\section{Materials and Methods}

Materials

$\mathrm{AuCl}_{3} \cdot \mathrm{HCl} \cdot 4 \mathrm{H}_{2} \mathrm{O}$ was purchased from Shanghai No. 1 Reagent Factory, China. HEPES was generously provided by Nanjing SunShine Biotechnology Co., Ltd., China. Dulbecco's modified Eagle's medium (DMEM), fetal bovine serum (FBS), and trypsin-EDTA were purchased from Gibco, USA. MTT and 2,7-dichlorofluorescein diacetate (DCFH-DA) were purchased from Beyotime Institute of Biotechnology, China. All other chemical reagents were of analytical grade. Milli-Q water with electrical resistance of $18.2 \mathrm{M} \Omega \mathrm{cm}$ was used in all of our experiments.

\section{Preparation of HEPES-Prepared and Citric-Prepared GNPs}

$\mathrm{AuCl}_{3} \cdot \mathrm{HCl} \cdot 4 \mathrm{H}_{2} \mathrm{O}$ and HEPES solutions need to be filtered through $0.22-\mu \mathrm{m}$ microporous membrane filters prior to use. HEPES-prepared GNPs were prepared by adding HEPES to boiling $\mathrm{AuCl}_{3} \cdot \mathrm{HCl} \cdot 4 \mathrm{H}_{2} \mathrm{O}$ aqueous solution with different concentrations corresponding to the HEPES/Au molar ration of 1:10, 2:10, 4:10, 8:10, and 20:10 and stirred to be homogenous in order to form a stable mixed liquor.

Citric-prepared GNPs were prepared according to the report of Gu et al. [20]. Briefly, they were prepared by adding trisodium citrate solution to boiling $\mathrm{AuCl}_{3} \cdot \mathrm{HCl} \cdot 4 \mathrm{H}_{2} \mathrm{O}$ aqueous solution at the molar ratio of 2:1. The final volume of citric-prepared GNPs was adjusted with de-ionized water, and the concentration was $100 \mathrm{ppm}(0.1 \mu \mathrm{g} / \mathrm{mL}$ of gold $)$.

UV-Vis Measurement

UV-vis measurement was performed with UV2450 UV-vis spectrophotometer (Shimadzu, Kyoto, Japan) in the absorbance mode and the range from 400 to $650 \mathrm{~nm}$ using deionized water as the reference. The fresh HEPES-prepared and citric-prepared GNP solution without any further treatment was used as samples for UV-vis measurement using deionized water as a standard for background correction.

Transmission Electron Microscopy Measurement

TEM measurements were performed in JEM-200 CX microscope (JEOL, Tokyo, Japan) operated at an accelerating voltage of $80 \mathrm{kV}$. The samples for TEM measurements were prepared by placing a drop of GNP solution onto copper grids and drying at room temperature. Micrographs at various positions were taken, and the sizes of the preferentially present particles were estimated based on several micrographs. The particle sizes were analyzed by software Image-pro plus 6.0.

\section{FT-IR Analysis and Zeta Potential Measurement}

FT-IR spectra were obtained using Nicolet Avater 370 spectrophotometer (Thermo, MA, USA). All the colloidal nanoparticles for FT-IR measurement were prepared by evaporating under reduced pressure to remove the solvent. The resulting precipitate was collected by centrifuging at 4,000 rpm for $5 \mathrm{~min}$, rinsing periodically with alcohol, and then drying under vacuum. The samples were then mixed with potassium bromide, ground, and pelletized. Liquid specimen was prepared by coating a thin film of the pure ionic liquid on a $\mathrm{KBr}$ disk. 
The zeta potential of HEPEs-prepared GNPs was measured on a Zetasizer (EEN3690, Malvern Instruments, Ltd., UK). GNPs were suspended in double-distilled water and placed in an electrophoretic cell $(3 \mathrm{~mL})$ and the electrophoretic mobility was measured at room temperature.

\section{Biosafety Studies of In Vivo Applications}

Specific pathogen free Spleen-Deficiency (SD) rats of both genders (male, 200-215 g; female, 175-190 g) were obtained from the Experimental Animal Center of Nantong University. For every cage about three rats were housed under controlled environmental conditions $\left(22 \pm 3{ }^{\circ} \mathrm{C}\right.$, $45-70 \%$ relative humidity, and a $12 \mathrm{~h} \mathrm{light/dark} \mathrm{cycle).} \mathrm{All} \mathrm{animal} \mathrm{experiments} \mathrm{were} \mathrm{approved}$ by the Care Committee of Nantong University which conducted the ethical review of the experiments.

The methods of acute toxicity studies were based on the OECD Guidelines for the Testing of Chemicals, "ROS Results". The limited dose level of HEPES-prepared and citric-prepared GNPs at least $500 \mu \mathrm{g} / \mathrm{kg}$ body weight/day (adjusting the final volume with the animal weight for the given dose) was administered by gavage to six male and six female rats.

The surviving rats were then sacrificed by decapitation under ketamine anesthesia. All organs were observed macroscopically, and selected vital organs (including liver, spleen and kidney) were excised, blotted, and weighed. Organ coefficient was also measured. Hematoxylin-eosin staining was performed as described [7]. Histological examination was performed under light microscopy (Olympus CKX 41).

\section{Biocompatibility Studies of In Vitro Applications}

Cytotoxicity was evaluated by MTT assay using well-grown passage L929 mouse fibroblasts under the standard ISO 10993.5-1999. The cells were seeded in $25-\mathrm{cm}^{2}$ plastic culture flasks and cultured in DMEM that was supplemented with $10 \%$ fetal bovine serum. The medium was renewed every day, and cultures were maintained in a 5-\% $\mathrm{CO}_{2}$ incubator at $37^{\circ} \mathrm{C}$. After $80 \%$ confluence was reached, the cells were detached using $0.25 \%$ trypsin-EDTA and transferred.

L929 mouse fibroblasts were seeded into 96-well plates at a density of $1 \times 10^{4}$ cells per well. After $12 \mathrm{~h}$ of preculture, the cells were treated with different concentrations of HEPESprepared and citric-prepared GNPs $(5.0,10.0,15.0,20.0$, and $25.0 \mathrm{ppm})$. Gold-free wells cultured under the same conditions were used as controls.

After 24 and $48 \mathrm{~h}$ of treatment, $20 \mu \mathrm{L}$ MTT solution $(5 \mathrm{mg} / \mathrm{mL})$ was added to each well and incubated for $4 \mathrm{~h}$ in a humidified atmosphere $\left(37{ }^{\circ} \mathrm{C}, 5 \% \mathrm{CO}_{2}\right)$ to form formazan crystals. Then, the culture medium was removed, and $150 \mu \mathrm{L}$ dimethyl sulfoxide was added to each well to solubilize formazan dye. After $15 \mathrm{~min}$, the absorbance was measured at $490 \mathrm{~nm}$ in a microplate reader.

\section{Measurement of ROS Production}

The amount of ROS was utilizing the DCFH-DA assay as previously described [13]. After $24 \mathrm{~h}$ of treatment, cells were seeded into 96-well plates at a density of 20,000 cells per well. After recovery, cells were washed twice with Hank's balanced salt solution and incubated with $50 \mu \mathrm{M}$ DCFH-DA for $15 \mathrm{~min}$. Cells were then washed with Hank's balanced salt solution, with non-treated cells used as negative controls and with rosup-treated cells employed as positive controls. After exposure to the chemicals, the fluorescence intensity of a total of 5,000 cells was measured by the FACS Calibur and Cell Quest Pro software (BD Biosciences). 
Statistical Analysis

The results were presented with mean \pm SD and analyzed using SPSS 13.0. Experimental data were examined for equal variance and normal distribution prior to statistical analysis. One-way analysis of variance (ANOVA) was used for statistical analysis. Values were considered significantly different if $P<0.05$.

\section{Results}

UV-Vis Spectra and TEM Results

Figure 1a illustrates the UV-vis spectra of GNPs prepared with different HEPES/Au ratios. The absorbance peak at the wavelength of around $525 \mathrm{~nm}$ in UV-vis spectra implied formation of GNPs. With the HEPES/Au ratio varied from 1:10 to 20:10, it could be found that the peak heights increased gradually. Figure 1b shows TEM images of GNPs prepared with the HEPES/Au ratio of $2: 10,4: 10$, and 8:10. There were some HEPESprepared GNPs with triangle or polygon forms at the HEPES/Au ratio of 2:10. Sizes of GNPs were more uniform at the HEPES/Au ratio of 4:10 compared with citricprepared GNPs (Fig. 1d). The inset shows the distribution diameter of the GNPs obtained. Table 1 lists the differences of the corresponding $\lambda_{\max }$ of GNPs prepared with citrate and HEPES with different HEPES/Au ratios. Such results revealed that smaller GNPs with narrow and uniform distribution could be obtained at the HEPES/ $\mathrm{Au}$ ratio of 4:10. By comparison, citrate-prepared GNPs showed a wider distribution at the $\mathrm{Na}_{3}$ citrate/Au molar ratio of 20:10.

Since uniform GNPs could be obtained at the HEPES/Au ratio of 4:10, the as-prepared GNPs were then applied for in vitro and in vivo experiments.

\section{FT-IR and Zeta Results}

Figure 2a shows the FT-IR spectra of pure HEPES and powders of HEPES-prepared GNPs at the HEPES/Au ratio of 4:10. It could be found that both HEPES-prepared GNP powders and pure HEPES exhibited two IR bands at 3,134 and $1,338 \mathrm{~cm}^{-1}$, which could be attributed to the stretching bands of $\mathrm{C}-\mathrm{H}$ and $\mathrm{C}-\mathrm{O}$, respectively. We can infer that the HEPES formed in the part of the prepared GNPs. Figure 2a also shows the difference of $v \mathrm{~S}-\mathrm{H}$ vibration band at $2,538 \mathrm{~cm}^{-1}$ between the GNPs and HEPES, which suggests that the $\mathrm{S}-\mathrm{H}$ bond is broken upon binding to the gold particle surface to form the bond of $\mathrm{S}-\mathrm{Au}$ [21]. The function of thiol groups in HEPES is to prevent $\mathrm{Au}^{(0)}$ particles from aggregating and control the growth of nanoparticle in aqueous media [15].

Since the size of HEPES-prepared GNPs was greater than $10 \mathrm{~nm}, 50 \mathrm{ppm}$ suspension of gold nanoparticles was used for the measurement of zeta potential. As can be seen in Fig. 2b, the gold nanoparticles displayed the highest count with a $\zeta$ value of $-31.7 \mathrm{mV}$, indicating a moderately negative charge on the surface of the nanoparticles.

\section{Biosafety Studies}

The study of acute oral toxicity includes mortality, body weight variation, and histological examination. At a dose of $500 \mu \mathrm{g} / \mathrm{kg}$, all treated rats grew normally and survived in the observation period. It could be found from Table 2 that the mean body gain decreased in the 

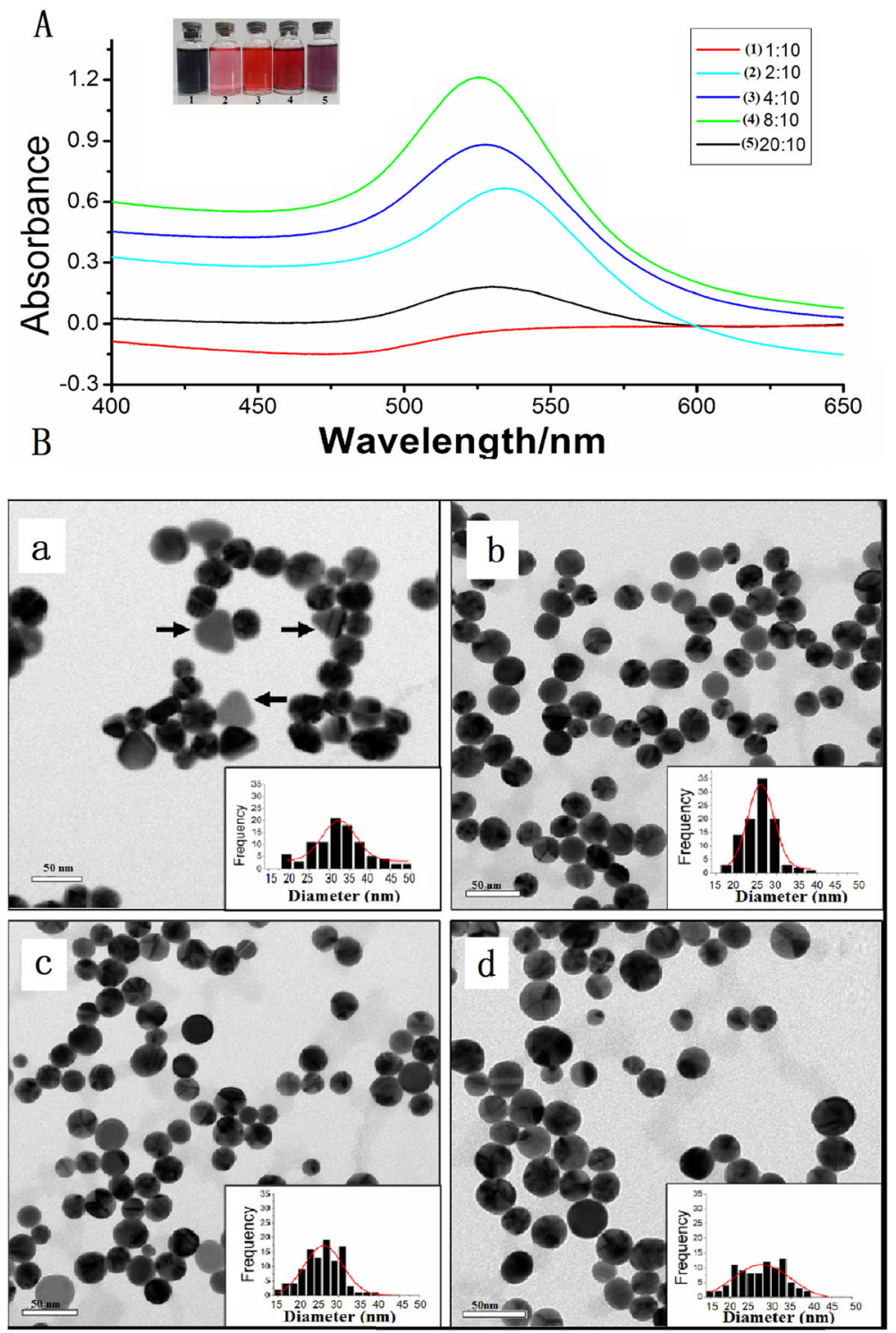

Fig. 1 Characterization of HEPES-prepared and citric-prepared GNPs. a UV-vis spectra and photograph of several vials containing synthesis of HEPES-prepared GNPs by different molar ratios of HEPES/Au: (1) 1:10, (2) 2:10, (3) 4:10, (4) 8:10, (5) 20:10. b TEM images and histograms of size distribution of HEPES-prepared GNPs (a: 2:10, b 4:10, c 8:10) and citric-prepared GNPs (d). The arrows show the triangle or polygon of HEPEsprepared GNPs. The sphere GNPs with narrow uniform distribution could be obtained with the HEPES/Au ratio of $4: 10$

group fed with citric-prepared GNPs (male, $P<0.05$; female, $P<0.01$ ) after the test. By comparison, no significant change was observed on the mean body gain for the group fed with HEPES-prepared GNPs. As shown in Table 3, organ coefficients showed no considerable change in both sexes except the liver dosed with citric-prepared GNPs (compared with the control group, male, $P<0.05$; female, $P<0.01$ ). The organ coefficients of liver of both male 
Table 1 UV-vis absorption peak position $\left(\lambda_{\max }\right)$ and the average particle diameters (ds) of HEPES-prepared GNPs changed with different molar ratio of HEPES/Au

\begin{tabular}{lllll}
\hline & Citric-prepared GNPs (citrate/Au) & \multicolumn{3}{l}{ HEPES-prepared GNPs (HEPES/Au) } \\
\cline { 3 - 5 } & $20: 10$ & $2: 10$ & $4: 10$ & $8: 10$ \\
\hline$\lambda_{\max (\mathrm{nm})}$ & 524.2 & 527.3 & 521.4 & 523.7 \\
ds $(\mathrm{nm})$ & $27.42 \pm 5.84$ & $32.37 \pm 6.59$ & $26.01 \pm 4.00^{\mathrm{a}}$ & $26.33 \pm 5.21$ \\
\hline
\end{tabular}

${ }^{\text {a }} P<0.01$ when compared with citric-prepared GNPs

and female rats in citric-prepared GNP group were significantly higher than HEPES-prepared GNP group $(P<0.05)$.

Histological examination of various tissues was carried out for other toxic effects. All of the specimens harvested during necropsy had no noteworthy reorganization in their histological structure. Rats exposed to HEPES-prepared and citric-prepared GNPs showed no tissue damage in any section from kidney and spleen except liver. As shown in Fig. 3, inflammatory

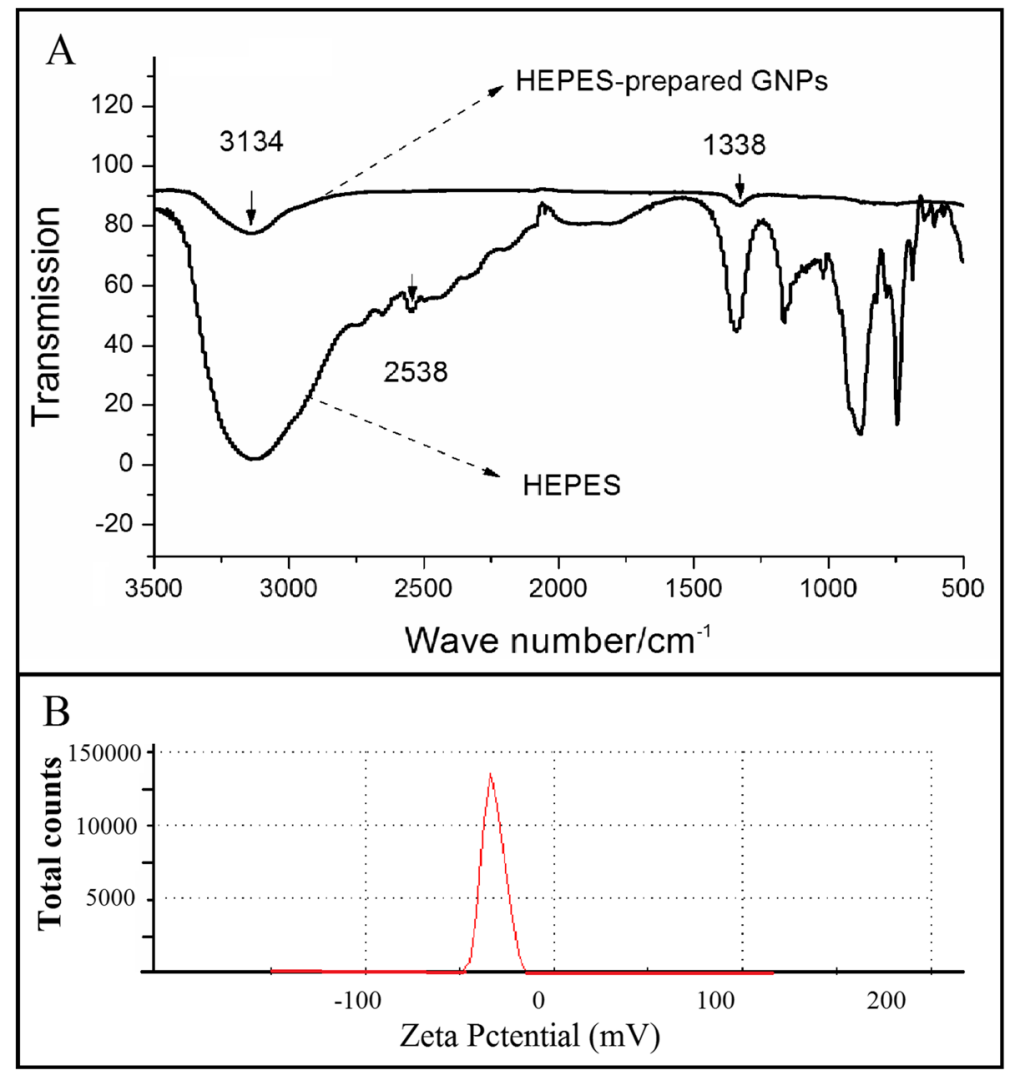

Fig. 2 a FT-IR spectra of the HEPES-prepared GNPs powder and pure HEPES. b The zeta potential distribution of R-GNPs in aqueous medium. Both HEPES-prepared GNP powders and pure HEPES exhibited two IR bands at 3,134 and $1,338 \mathrm{~cm}^{-1}$. The powders of HEPES-prepared GNPs show no detectable characteristic bands of S-H vibration in the range of 2,500-2,600 $\mathrm{cm}^{-1}$. The gold nanoparticles displayed the highest count with a $\zeta$ value of $-31.7 \mathrm{mV}$, indicating a moderately negative charge on the surface of the nanoparticles 
Table 2 Body weight of rats in HEPES-prepared and citric-prepared GNPs acute oral toxicity study at a limited dose of $500 \mu \mathrm{g} / \mathrm{kg}$ bw

\begin{tabular}{|c|c|c|c|c|c|c|}
\hline \multirow[t]{2}{*}{ Parameter } & \multicolumn{3}{|l|}{ Male } & \multicolumn{3}{|l|}{ Female } \\
\hline & Control & $\begin{array}{l}\text { Citric- } \\
\text { prepared } \\
\text { GNPs }\end{array}$ & $\begin{array}{l}\text { HEPES- } \\
\text { prepared } \\
\text { GNPs }\end{array}$ & Control & $\begin{array}{l}\text { Citric- } \\
\text { prepared } \\
\text { GNPs }\end{array}$ & $\begin{array}{l}\text { HEPES- } \\
\text { prepared } \\
\text { GNPs }\end{array}$ \\
\hline $\begin{array}{l}\text { Initial body } \\
\text { weight (g) }\end{array}$ & $206.48 \pm 4.97$ & $206.54 \pm 4.78$ & $205.84 \pm 4.32$ & $181.40 \pm 4.53$ & $181.02 \pm 4.87$ & $182.38 \pm 4.43$ \\
\hline $\begin{array}{l}\text { Final body } \\
\text { weight }(\mathrm{g})\end{array}$ & $260.38 \pm 10.07$ & $245.14 \pm 10.59^{\mathrm{a}, \mathrm{c}}$ & $258.87 \pm 9.46$ & $229.60 \pm 7.71$ & $214.24 \pm 10.43^{\mathrm{a}, \mathrm{c}}$ & $229.44 \pm 9.89$ \\
\hline $\begin{array}{l}\text { Body weight } \\
\text { gain }(\mathrm{g})\end{array}$ & $53.90 \pm 7.51$ & $38.60 \pm 5.81^{\mathrm{a}, \mathrm{c}}$ & $53.04 \pm 6.28$ & $48.20 \pm 6.14$ & $33.22 \pm 5.57^{\mathrm{b}, \mathrm{d}}$ & $47.06 \pm 12.64$ \\
\hline
\end{tabular}

Values were means $\pm \mathrm{SD}$ of six mice in each group

${ }^{\text {a }} P<0.05$ when compared with the control group in the same sex rats

${ }^{\mathrm{b}} P<0.01$ when compared with the control group in the same sex rats

${ }^{\mathrm{c}} P<0.05$ when citric-prepared GNPs compared to the same concentrations of HEPES-prepared GNPs group

${ }^{\mathrm{d}} P<0.01$ when citric-prepared GNPs compared to the same concentrations of HEPES-prepared GNPs group

liver injury could be observed in the group treated with citric-prepared treated GNPs but not with HEPES-prepared GNPs.

\section{Biocompatibility Studies}

Figure 4a illustrated the MTT reduction reaction of L929 cells cultured for 24 and $48 \mathrm{~h}$ in the medium supplemented with HEPES-prepared or citric-prepared GNPs at different concentrations, respectively. The lines mean the OD of control groups at 24 and $48 \mathrm{~h}$. The L929 fibroblasts co-cultured with 5.0 and 10.0 ppm HEPES-prepared GNPs presented a high proliferation rate compared to the control group throughout the culture time ( $24 \mathrm{~h}, P<0.05$; $48 \mathrm{~h}, P<0.01)$. Furthermore, cell viability was higher for 5.0 and $10.0 \mathrm{ppm}$ HEPES-prepared GNPs than citric-prepared GNPs $(P<0.05)$. As GNP concentration increased, L929 cell proliferation was inhibited in varying degrees. Higher doses (20.0 and $25.0 \mathrm{ppm})$ of citric-

Table 3 Organ coefficients of both male and female rats in citric-prepared and HEPES-prepared GNPs group

\begin{tabular}{|c|c|c|c|c|c|c|}
\hline \multirow[t]{2}{*}{ Parameter } & \multicolumn{3}{|l|}{ Male } & \multicolumn{3}{|l|}{ Female } \\
\hline & Control & $\begin{array}{l}\text { Citric- } \\
\text { prepared } \\
\text { GNPs }\end{array}$ & $\begin{array}{l}\text { HEPES- } \\
\text { prepared } \\
\text { GNPs }\end{array}$ & Control & $\begin{array}{l}\text { Citric- } \\
\text { prepared } \\
\text { GNPs }\end{array}$ & $\begin{array}{l}\text { HEPES- } \\
\text { prepared } \\
\text { GNPs }\end{array}$ \\
\hline Liver (g/100 g bw) & $3.46 \pm 0.28$ & $3.86 \pm 0.23^{\mathrm{a}, \mathrm{c}}$ & $3.48 \pm 0.29$ & $2.78 \pm 0.26$ & $3.42 \pm 0.27^{\mathrm{b}, \mathrm{c}}$ & $2.98 \pm 0.25$ \\
\hline Kidneys (g/100 g bw) & $0.94 \pm 0.12$ & $0.93 \pm 0.08$ & $0.90 \pm 0.07$ & $0.74 \pm 0.10$ & $0.82 \pm 0.09$ & $0.81 \pm 0.10$ \\
\hline Heart (g/100 g bw) & $0.41 \pm 0.02$ & $0.43 \pm 0.04$ & $0.40 \pm 0.01$ & $0.35 \pm 0.02$ & $0.36 \pm 0.02$ & $0.34 \pm 0.01$ \\
\hline Spleen $(\mathrm{g} / 100 \mathrm{~g}$ bw) & $0.27 \pm 0.05$ & $0.26 \pm 0.05$ & $0.25 \pm 0.06$ & $0.25 \pm 0.03$ & $0.28 \pm 0.03$ & $0.24 \pm 0.05$ \\
\hline
\end{tabular}

Values were means $\pm \mathrm{SD}$ of six mice in each group

${ }^{\text {a }}$ Significantly different with the control group in the same sex at $P<0.05$

${ }^{\mathrm{b}}$ Very significantly different from control group, $P<0.01$

${ }^{\mathrm{c}}$ Significantly different with HEPES-prepared GNPs group in the same sex at $P<0.05$ 


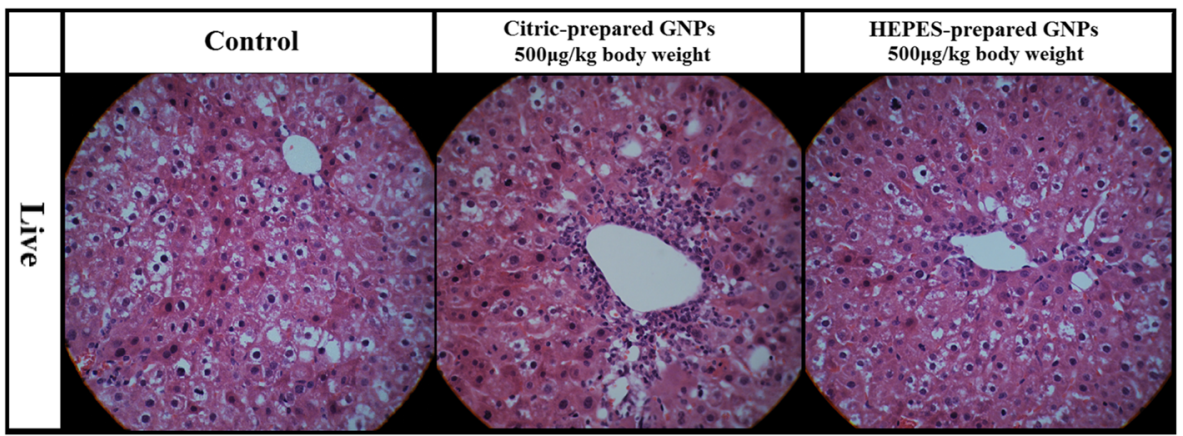

Fig. 3 Histological analysis of various organs after $500 \mu \mathrm{g} / \mathrm{kg}$ body weight citric-prepared and HEPES-prepared GNP treatment. Slight inflammation was observed in pathological sections of liver tissue in the citric-prepared GNP group $(\mathrm{H} \& \mathrm{E}, \times 200)$

prepared GNPs (48 h) showed a thorough inhibition on cell proliferation. Cell viability at $48 \mathrm{~h}$ was significantly higher with 20.0 and 25.0 ppm HEPES-prepared GNPs than those with citric-prepared GNPs $(P<0.01)$, although the HEPES GNPs also inhibit cell proliferation at 20 and $25 \mathrm{ppm}$ at $48 \mathrm{~h}$.

One noticeable effect on biocompatibility was different cell shapes and morphology in the monolayer. It could be found from Fig. $4 \mathrm{~b}$ that the shapes of cells treated with $20.0 \mathrm{ppm}$ HEPES-prepared GNPs were triangle, spindle, and extended pseudopodia to adhere to the culture flasks. By comparison, only a few cells treated with 20.0 ppm citric-prepared GNPs could survive and maintain the triangle and spindle shape.
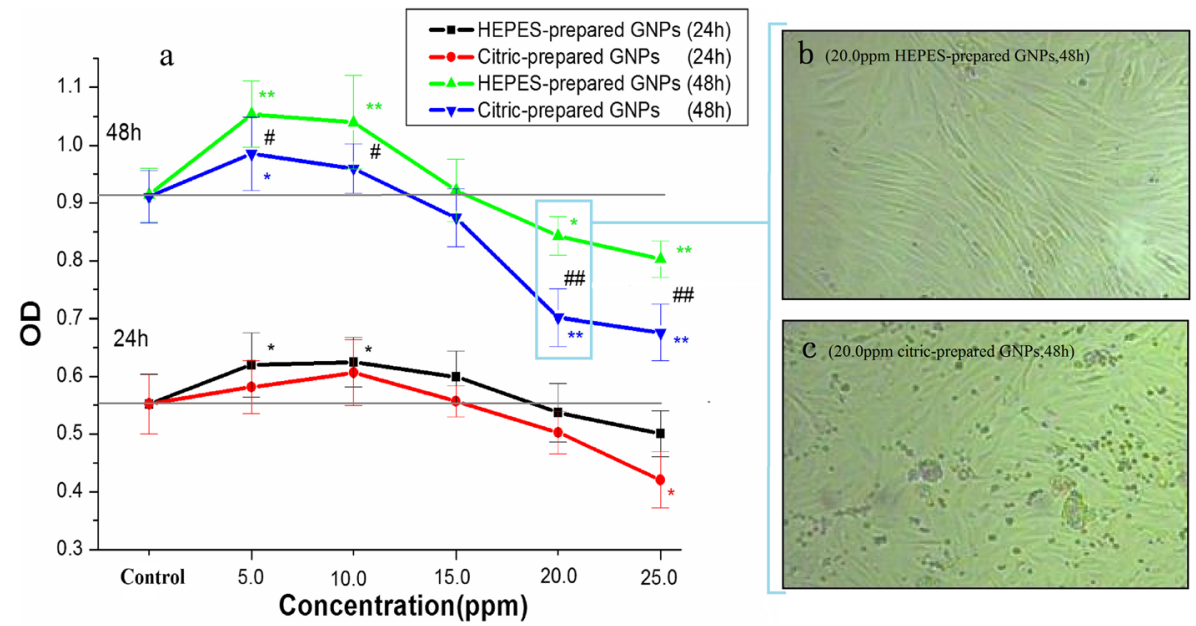

Fig. 4 The cytotoxicity test of GNPs was performed using mouse fibroblast cells, which were treated with different concentrations of HEPES-prepared and citric-prepared GNPs (0 (as the control), 5.0, 10.0, 15.0, 20.0, and $25.0 \mathrm{ppm}$ ). After 24 and $48 \mathrm{~h}$, the cells' vitality was tested by MTT assay. The absorbance was measured at the wavelength of $490 \mathrm{~nm}$ in a microplate reader. The growth curve of MTT reduction reaction results (a), the cell proliferation and morphology of 20.0 ppm HEPES-prepared GNPs group (b) and 20.0 ppm citric-prepared GNPs (c) at $48 \mathrm{~h}$. Asterisk significantly different from control group, $P<0.05$; double asterisks very significantly different from control group, $P<0.01$. Number sign significantly different compared to the same concentrations of GNP group, $P<0.05$; number sign pair very significantly different from the same concentrations of GNP group, $P<0.01$ 


\section{ROS Results}

In the following, we examined the dependence of ROS production of cells on treatment of HEPES-prepared and citric-prepared GNPs (Fig. 5). It could be found that the amount of ROS with HEPES-prepared and citric-prepared GNPs at the concentration of 5.0 and $10.0 \mathrm{ppm}$ were similar to the negative group. With the increase of GNP concentration to $15.0 \mathrm{ppm}$, there were more ROS generated in the cells treated with citric-prepared GNPs than with HEPES-prepared GNPs $(P<0.01)$. Although the amounts of ROS increased with the increase of GNP concentration, they were significantly lower than the positive control (Fig. 5, inset). Overall, there were more ROS generated in citric-prepared GNPs than in HEPES-prepared GNPs.

\section{Discussion}

In this study, we prepared GNPs with HEPES, which is a popular buffer solution extensively used in biological laboratories for cell and tissue culture [16]. HEPES has a pKa close to physiological conditions $(\mathrm{pH}=7.5)$, maximum aqueous solubility, low permeability to cell membrane, and high chemical stability [17]. It has been reported that a simple one-pot synthesis of three-dimensional gold nanocrystals with multi-tips could be fabricated by adding $\mathrm{HAuCl}_{4}$ solution into HEPES without shaking and heating [17], and HEPES is serving as both reducing and shape-directing agent. GNPs with polygon forms could also be prepared by mixing HEPES with an aqueous solution of $6 \mathrm{ppm} \mathrm{Au} \mathrm{(III)} \mathrm{at} \mathrm{room} \mathrm{temperature} \mathrm{[16].} \mathrm{Herein}$ we mixed $\mathrm{HEPES}$ to boiling $\mathrm{AuCl}_{3} \cdot \mathrm{HCl} \cdot 4 \mathrm{H}_{2} \mathrm{O}$ aqueous solution and optimized the conditions in order to obtain sphere GNPs to improve their biosafety and biocompatibility [18]. Our results showed that GNPs with sphere shape, uniform distribution, and well stability could be

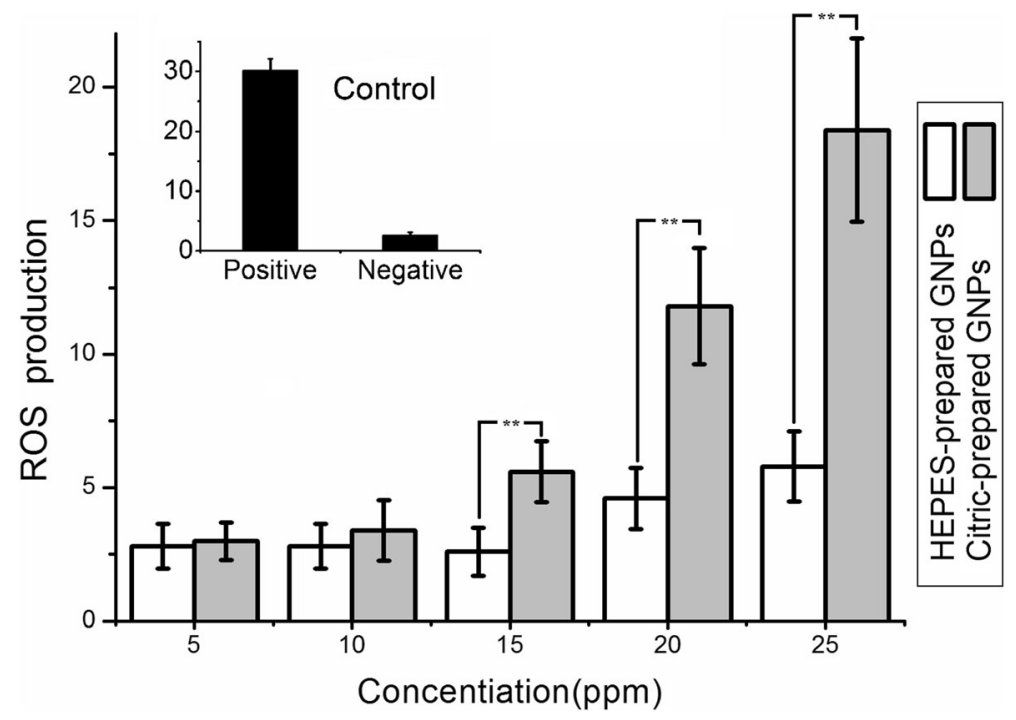

Fig. 5 Amount of ROS produced relative to L929 cells treated with different concentrations of HEPES-prepared and citric-prepared GNPs $(5.0,10.0,15.0,20.0$, and $25.0 \mathrm{ppm})$. The insets show relative ROS produced in cells following positive and negative controls. Data represent mean $\pm \mathrm{SD}(n=5)$. Double asterisks very statistically significantly different between the citric-prepared GNP treated group and HEPES-prepared GNP treated group, $P<0.01$ 
achieved at the HEPES/Au ratio of 4:10, which indicated that the number of thiol groups in ionic liquid plays an important role in the stabilization of gold nanoparticles. The interaction between gold atoms and thiol groups in HEPES could be further investigated by FT-IR spectra.

It is believed that the mechanism of GNPs formation in our approach was similar to that in previous reports [16]. The surface of GNPs could be modified by the thiol group in HEPES because of the strong connection between the gold surface and thiol/amine [19]. FT-IR results confirmed this step with the stretching band of methylene, which could be ascribed to the modification of the thiol group on the surface of gold clusters [20]. We think that the boiling process could facilitate the stabilization of GNPs and prevent the aggregation at higher temperature. The process of GNP formation was further proved by the characteristic bands in surface plasmon resonance (SPR) [22]. The moderate value of zeta potential might also reflect that the $\mathrm{S}-\mathrm{H}$ bond is broken upon binding to the gold particle surface to form the bond of S-Au and the electrostatically stable form of HEPES-prepared GNPs.

The harmfulness of GNPs to mammalian cells was thought to depend on their sizes or residual hazardous chemicals. Pan et al. reported that GNPs of $1-2 \mathrm{~nm}$ in size were more toxic than 15-nm gold colloids in the study of the cytotoxicity of TPPMS/TPPTS-modified gold nanoparticles [23]. Their results suggested that the sizes of GNPs might play dominant roles in the metabolism of nanoparticles [9]. Shukla et al. found that $\mathrm{Au}(0)$ nanoparticles were not cytotoxic and might become a suitable candidate for nanomedicine. The reason is that GNPs could reduce the production of reactive oxygen and nitrite species without eliciting secretion of proinflammatory cytokines TNF- $\alpha$ and IL1- $\beta$ [24].

We demonstrated that HEPES-prepared GNPs with a size of $26.01 \mathrm{~nm}$ exhibited improved biosafety compared with citric-prepared GNPs. For example, HEPES-prepared GNPs could not pass through the glomerular filtration of kidneys due to its size and negative electrostatic potential [25]. The bioaccumulation of HEPES-prepared GNPs may be because of their regulation in the reticulo-endothelial system, which is involved in the uptake and metabolism of exogenous nanoparticles in these tissues [26]. Interestingly, there were more viable cells in the groups treated with 5.0 and 10.0 ppm HEPES-prepared GNPs (48 h) than in the control, which was consistent with our previous study: a concentration of $5.0 \mathrm{ppm}$ gold nanoparticle has the best effect on the promotion of cell growth [27]. This may be due to the fact that the small amount of gold nanoparticles could enter the cell and enhance DNA synthesis. Our in vitro results confirmed that HEPES-prepared GNPs showed better biocompatibility at the concentration of 5.0 to $10.0 \mathrm{ppm}$. Although GNPs have been considered bioinert and therapeutically used, GNPs might change the hematology and blood chemistry, indicating potential damage on liver and kidney as well as histological disorders, such as apoptosis and inflammation in various organs $[6,7,28]$. While administrated to rats, citric-prepared GNPs could disappear from the circulation and localized in the tissues, primarily the liver [26]. Our results indicate that the administration of HEPES-prepared GNPs could not induce extensive inflammation on rats, which are superior to citric-prepared GNPs. In liver (heavy metal detoxification organ), there were some inflammatory cells after injection of citric-prepared GNPs. It is probably caused by the reducing agent citric acid. Such experimental results agreed with the previous report from Lasagna-Reeves et al. [3].

The MTT and ROS assay were utilized to characterize the effect of HEPES-prepared and citric-prepared GNPs on L929 cell proliferation. Oxidative stress, which includes ROS generation, is currently the most popular theory in the study of nanoparticle toxicity [29]. Oxidative stress has been shown to occur in cells following exposure to gold nanoparticles [30]. Citric-prepared GNP was more effective in increasing ROS compared to the negative control as the concentration rises above $20.0 \mathrm{ppm}$, which was consistent with the fact that the proliferation of cells was significantly inhibited by citric-prepared GNPs at 20.0- and 25.0-ppm 
concentrations. Some cells began to shrink and disintegrate, which might be due to the increased ROS levels.

Interestingly, it was found that ROS production slightly decreased in groups treated with HEPES-prepared GNPs at the low concentrations. It might be possible that agents binding to the AuNPs through $\mathrm{Au}-\mathrm{S}$ bonds could decrease the catalytic activity of the AuNPs. Pan et al. demonstrated that several thiol-containing antioxidants could significantly reduce their toxic effects [29]. MTT and ROS results showed that cytotoxicity might depend on both the concentration of GNPs and the type of reducing agent used.

\section{Conclusions}

In summary, our investigation demonstrated that HEPES could get a better reducing agent and stabilizer for GNPs from the view of "biocompatible". HEPES-prepared GNPs showed better biosafety and biocompatibility than citric-prepared GNPs under both in vivo and in vitro circumstances. Preparation of GNPs with HEPES is convenient for biomedical applications. This study not only demonstrated the key fact of reducing agent but also accumulated more evidence for the study of biosafety and biocompatibility of nanoparticles.

Acknowledgments This work was supported by the National Natural Science Foundation of China (Nos. 21175075, 21075070, and 21375066), the Natural Science Foundation of Jiangsu Province of China (Nos. BK2011047, BK2012651, and BK2012652), Social Development Item of Nantong City (Nos. BK 2013028 and BK 2012058), and the Priority Academic Program Development of Jiangsu Higher Education Institutions (PAPD) for their support of this research.

\section{References}

1. He, C., Zhang, L., Wang, H., Zhang, F., \& Mo, X. (2009). Physical-chemical properties and in vitro biocompatibility assessment of spider silk, collagen and polyurethane nanofiber scaffolds for vascular tissue engineering. Nano Biomedicine and Engineering, 1(1), 80-88.

2. Zhang, X., Pan, B., Wang, K., Ruan, J., Bao, C., Yang, H., \& Cui, D. (2010). Electrochemical property and cell toxicity of gold electrode modified by monolayer PAMAM encapsulated gold nanorods. Nano Biomedicine and Engineering, 2(3), 182-188.

3. Lasagna-Reeves, C., Gonzalez-Romero, D., Barria, M. A., Olmedo, I., Clos, A., Sadagopa Ramanujam, V. M., \& Soto, C. (2010). Bioaccumulation and toxicity of gold nanoparticles after repeated administration in mice. Biochemical and Biophysical Research Communications, 393(4), 649-655.

4. Doron, A., Katz, E., \& Willner, I. (1995). Organization of Au colloids as monolayer films onto ITO glass surfaces: application of the metal colloid films as base interfaces to construct redox-active monolayers. Langmuir, 11(4), 1313-1317.

5. Zhang, X. D., Wu, D., Shen, X., Liu, P. X., Yang, N., Zhao, B., \& Fan, F. Y. (2011). Size-dependent in vivo toxicity of PEG-coated gold nanoparticles. International Journal of Nanomedicine, 6, 2071.

6. Cho, W. S., Cho, M., Jeong, J., Choi, M., Cho, H. Y., Han, B. S., \& Jeong, J. (2009). Acute toxicity and pharmacokinetics of 13 nm-sized PEG-coated gold nanoparticles. Toxicology and Applied Pharmacology, 236(1), 16-24.

7. Chen, Y. S., Hung, Y. C., Liau, I., \& Huang, G. S. (2009). Assessment of the in vivo toxicity of gold nanoparticles. Nanoscale Research Letters, 4(8), 858-864.

8. Zhang, X. D., Wu, D., Shen, X., Liu, P. X., Fan, F. Y., \& Fan, S. J. (2012). In vivo renal clearance, biodistribution, toxicity of gold nanoclusters. Biomaterials, 33(18), 4628-4638.

9. Hochstim, C. J., Choi, J. Y., Lowe, D., Masood, R., \& Rice, D. H. (2010). Biofilm detection with hematoxylin-eosin staining. Archives of Otolaryngology-Head \& Neck Surgery, 136(5), 453-456.

10. Sieminski, A. L., \& Gooch, K. J. (2000). Biomaterial-microvasculature interactions. Biomaterials, 21(22), 2233-2241. 
11. Semmler-Behnke, M., Kreyling, W. G., Lipka, J., Fertsch, S., Wenk, A., Takenaka, S., \& Brandau, W. (2008). Biodistribution of 1.4- and 18-nm gold particles in rats. Small, 4(12), 2108-2111.

12. Sadauskas, E., Wallin, H., Stoltenberg, M., Vogel, U., Doering, P., Larsen, A., \& Danscher, G. (2007). Kupffer cells are central in the removal of nanoparticles from the organism. Particle and Fibre Toxicology, $4(10), 10$.

13. Simon, H. U., Haj-Yehia, A., \& Levi-Schaffer, F. (2000). Role of reactive oxygen species (ROS) in apoptosis induction. Apoptosis, 5(5), 415-418.

14. Liz-Marzán, L. M. (2006). Tailoring surface plasmons through the morphology and assembly of metal nanoparticles. Langmuir, 22(1), 32-41.

15. Daniel, M. C., \& Astruc, D. (2004). Gold nanoparticles: assembly, supramolecular chemistry, quantum-sizerelated properties, and applications toward biology, catalysis, and nanotechnology. Chemical Reviews, 104(1), 293-346.

16. Pham, T., Jackson, J. B., Halas, N. J., \& Lee, T. R. (2002). Preparation and characterization of gold nanoshells coated with self-assembled monolayers. Langmuir, 18(12), 4915-4920.

17. Sau, T. K., \& Murphy, C. J. (2004). Seeded high yield synthesis of short Au nanorods in aqueous solution. Langmuir, 20(15), 6414-6420.

18. Panchapakesan, B., Book-Newell, B., Sethu, P., Rao, M., \& Irudayaraj, J. (2011). Gold nanoprobes for theranostics. Nanomedicine, 6(10), 1787-1811.

19. Tiwari, P. M., Vig, K., Dennis, V. A., \& Singh, S. R. (2011). Functionalized gold nanoparticles and their biomedical applications. Nanomaterials, 1(1), 31-63.

20. De Jong, W. H., Hagens, W. I., Krystek, P., Burger, M. C., Sips, A. J., \& Geertsma, R. E. (2008). Particle size-dependent organ distribution of gold nanoparticles after intravenous administration. Biomaterials, 29(12), 1912-1919.

21. Gu, H. Y., Chen, Z., Sa, R. X., Yuan, S. S., Chen, H. Y., Ding, Y. T., \& Yu, A. M. (2004). The immobilization of hepatocytes on 24 nanometer sized gold colloid for enhanced hepatocytes proliferation. Biomaterials, 25 , 3445-3451.

22. Kim, K. S., Demberelnyamba, D., \& Lee, H. (2004). Size-selective synthesis of gold and platinum nanoparticles using novel thiol-functionalized ionic liquids. Langmuir, 20(3), 556-560.

23. Pan, Y., Leifert, A., Ruau, D., Neuss, S., Bornemann, J., Schmid, G., \& Jahnen-Dechent, W. (2009). Gold nanoparticles of diameter $1.4 \mathrm{~nm}$ trigger necrosis by oxidative stress and mitochondrial damage. Small, 5(18), 2067-2076.

24. Shukla, R., Bansal, V., Chaudhary, M., Basu, A., Bhonde, R. R., \& Sastry, M. (2005). Biocompatibility of gold nanoparticles and their endocytotic fate inside the cellular compartment: a microscopic overview. Langmuir, 21(23), 10644-10654.

25. Longmire, M., Choyke, P. L., \& Kobayashi, H. (2008) Clearance properties of nano-sized particles and molecules as imaging agents: considerations and caveats.

26. Abdelhalim, M. A. K., Al-Ayed, M. S., Alhomida, A. S., \& Moussa, S. A. A. (2013). Absorption optical density as a diagnostic tool for indicating the toxicity of gold nanoparticles. African Journal of Biotechnology, 10(73), 16654-16660.

27. Lu, S., Xia, D., Huang, G., Jing, H., Wang, Y., \& Gu, H. (2010). Concentration effect of gold nanoparticles on proliferation of keratinocytes.Colloids and. Surfaces B: Biointerfaces, 81(2), 406-411.

28. Terentyuk, G. S., Maslyakova, G. N., Suleymanova, L. V., Khlebtsov, B. N., Kogan, B. Y., Akchurin, G. G., \& Tuchin, V. V. (2009). Circulation and distribution of gold nanoparticles and induced alterations of tissue morphology at intravenous particle delivery. Journal of Biophotonics, 2(5), 292-302.

29. Nel, A., Xia, T., Mädler, L., \& Li, N. (2006). Toxic potential of materials at the nanolevel. Science, 311(5761), 622-627.

30. Pan, Y., Neuss, S., Leifert, A., Fischler, M., Wen, F., Simon, U., \& Jahnen-Dechent, W. (2007). Sizedependent cytotoxicity of gold nanoparticles. Small, 3(11), 1941-1949. 